\title{
Rogue waves in optically injected lasers: Origin, predictability, and suppression
}

\author{
Jordi Zamora-Munt, ${ }^{1,2}$ Bruno Garbin, ${ }^{3}$ Stéphane Barland, ${ }^{3}$ Massimo Giudici, ${ }^{3}$ Jose R. Rios Leite, ${ }^{4}$ \\ Cristina Masoller, ${ }^{1}$ and Jorge R. Tredicce ${ }^{3,5}$ \\ ${ }^{1}$ Departament de Fisica i Enginyeria Nuclear, Universitat Politecnica de Catalunya, Colom 11, E-08222 Terrassa, Barcelona, Spain \\ ${ }^{2}$ IFISC (CSIC-UIB), Campus Universitat Illes Balears, E-07122 Palma de Mallorca, Spain \\ ${ }^{3}$ Université de Nice Sophia Antipolis, Institut Non-Lineaire de Nice, UMR 6618, F-06560 Valbonne, France \\ ${ }^{4}$ Departamento de Fisica, Universidade Federal de Pernambuco, 50670-901 Recife, Brazil \\ ${ }^{5}$ Université de la Nouvelle Caledonie (UNC), Pole Pluridisciplinaire de la Matiere et de l'Environnement (PPME), \\ EA3325 Noumea, Nouvelle Caledonie
}

(Received 10 August 2012; published 7 March 2013)

\begin{abstract}
Rogue waves are devastating extreme events that occur in many natural systems, and a lot of work has focused on predicting and understanding their origin. In optically injected semiconductor lasers rogue waves are rare ultra-high pulses that sporadically occur in the laser chaotic output intensity. Here we show that these optical rogue waves can be predicted with long anticipation time, that they are generated by a crisis-like process, and that noise can be employed to either enhance or suppress their probability of occurrence. By providing a good understanding of the mechanisms triggering and controlling the rogue waves, our results can contribute to improve the performance of injected lasers and can also enable new experiments to test if these mechanisms are also involved in other natural systems where rogue waves have been observed.
\end{abstract}

DOI: 10.1103/PhysRevA.87.035802

PACS number(s): 42.65.Tg, 05.45.-a, 42.55.Px, 42.60.Mi

Extreme events are often catastrophic ones, such as tsunamis, earthquakes, supernovas, stock market crashes, etc. [1-5]. Ocean rogue waves, also referred to as freak waves, are several times the average height of surrounding waves and have steep, fast rising, and fast falling sides, like "a wall of water" [6-9]. They are a topic of intensive research as they can develop suddenly even in calm and apparently safe seas and have been responsible for several boat accidents, representing a major challenge for the design of off-shore platforms for the oil and gas industry.

In optics, Solli et al. [10] have shown that extremely broadband radiation can be generated from a narrow-band input, with a long-tailed distribution similar to that of ocean rogue waves. Since then, optical rogue waves have been observed in several systems [11-25], and their study has advanced the research in the field, in a way that has been compared to the introduction of optical systems to study chaos in the 1980s [26].

In lasers, rogue waves occurring in the form of giant intensity pulses capable of producing catastrophic optical damage have been observed in pump-modulated [19], Raman [20], mode-locked [21-23], and optically injected lasers [24]. In Ref. [24] the rogue waves were studied in the framework of a simple and deterministic model that exhibits two types of chaos: one in which rogue waves do not appear consistently and one in which they are relatively frequent [24]. Since a deterministic chaotic system possesses some correlation length, the rogue waves in the system should have some degree of predictability.

Here we show, experimentally and numerically, that these optical rogue waves can indeed be predicted with a long anticipation time as compared with the laser characteristic time scales. In addition, we show that an external crisis-like process $[27,28]$, in the form of the crossing of the attractor, developed from one fixed point, with the stable manifold of another fixed point, gives rise to an expanded attractor that supports trajectories with rogue waves. We also show that noise can be exploited for either enhancing or suppressing their probability of occurrence.

The experimental setup consists of a single-mode vertical cavity surface emitting semiconductor laser (VCSEL), which receives continuous-wave optical injection from an external grating tunable semiconductor laser [24]. An optical isolator ensures the coupling between the two devices is unidirectional. The time series $\left(8 \times 10^{6}\right.$ points recorded at $\left.20 \mathrm{GS} \mathrm{s}{ }^{-1}\right)$, are measured by an amplified photodetector (bandwidth DC $9.5 \mathrm{GHz}$ ) and recorded by a $6 \mathrm{GHz}$ bandwidth oscilloscope. Optical spectra measurement are obtained by a scanning Fabry Perot interferometer with $72 \mathrm{GHz}$ free spectral range and a finesse of about 110.

Optical rogue waves in the form of extreme intensity pulses were observed at certain injection conditions, and in order to test how predictable these rogue waves are, we consider a series of intensity pulses fulfilling the condition of rogue wave [29] and superpose the time-traces centered at the rogue wave; see Fig. 1(a). For comparison, Figs. 1(b)-1(d) present numerical time traces, obtained by simulating the model equations:

$$
\begin{gathered}
\dot{E}=\kappa(1+i \alpha)(N-1) E+i \Delta \omega E+\sqrt{P_{\text {inj }}}+\sqrt{D} \xi, \\
\dot{N}=\gamma_{n}\left(\mu-N-N|E|^{2}\right),
\end{gathered}
$$

where $E$ and $N$ are the complex field amplitude and the carrier density, $P_{\text {inj }}$ is the injection strength, $\Delta \omega=\omega_{s}-\omega_{m}$ is the detuning between the lasers, $D$ is the noise strength, and $\xi$ is a complex Gaussian white noise representing spontaneous emission. Other parameters are the field decay rate, $\kappa$, the carrier decay rate, $\gamma_{n}$, the line-width enhancement factor, $\alpha$, and the injection current, $\mu$. In all the simulations the laser intensity was low-pass filtered with a cutoff frequency of $9 \mathrm{GHz}$ to take into account the finite bandwidth of the experimental setup, and, unless otherwise explicitly stated, the parameters used are $P_{\text {inj }}=60 \mathrm{~ns}^{-2}, \Delta \nu=\Delta \omega / 2 \pi=$ $0.49 \mathrm{GHz}, \mu=2.2, \kappa=300 \mathrm{~ns}^{-1}, \gamma_{n}=1 \mathrm{~ns}^{-1}$, and $\alpha=3$. 

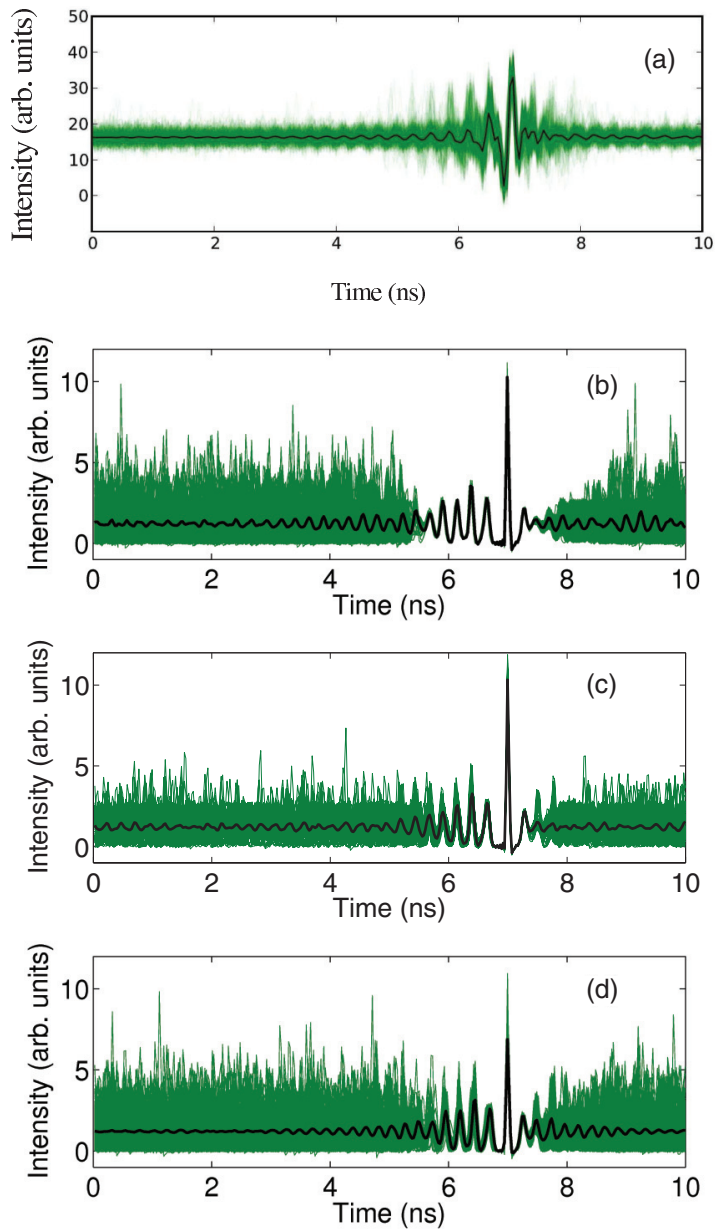

FIG. 1. (Color online) Superposition of time traces centered at the maximum of a rogue wave. (a) Experimental time series (500 rogue waves). (b) Deterministic simulations ( $D=0,459$ rogue waves). (c) Stochastic simulations ( $D=10^{-2} \mathrm{~ns}^{-1}, 53$ rogue waves). In panel (b) [in (c)] 20 (40) time traces were simulated of $415 \mu \mathrm{s}$ each. In panels (a), (b), and (c) the rogue wave threshold was defined as $\langle H\rangle+8 \sigma$ [29]. (d) As panel (b) but with the threshold defined as $\langle H\rangle+4 \sigma$. The black thick line displays the average of the time traces.

We observe that, for long times before and after a rogue wave, the superposition of traces generates just a wide band of intensity values. As the rogue wave approaches, the band shrinks, becoming almost a line. This observation is still valid when strong noise is added to the simulations [Fig. 1(c)]. These results show that just the knowledge of the intensity as a function of time is enough to predict the appearance of a rogue wave some time before it actually happens.

While a detailed study is left for future work, we remark here on three important aspects. First, the rogue waves have a duration of $0.1 \mathrm{~ns}$ and can be predicted up to $1.5 \mathrm{~ns}$ before they occur. Second, the prediction time increases with the threshold employed to define a rogue wave: A comparison of Figs. 1(b) and 1(d) shows that the higher the threshold is (i.e., the more "extreme" the rogue waves are), the longer the time in advance that they can be predicted. Third, the inclusion of noise significantly reduces the probability of occurrence of rogue waves, as seen by comparing the number of rogue waves
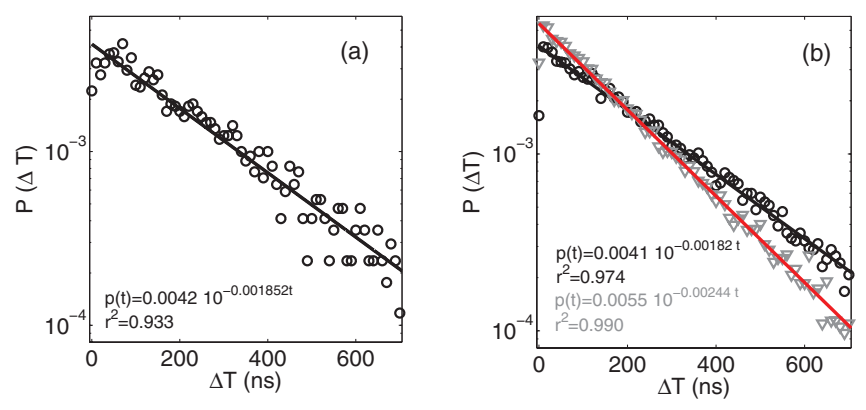

FIG. 2. (Color online) Probability density function of the interrogue-wave time intervals and the linear regression of the data (solid lines) for (a) experimentally observed time traces and (b) numerical simulations without noise (gray) and with noise ( $D=10^{-4} \mathrm{~ns}^{-1}$, black). Notice the very good agreement in the time scales when noise is included. The parameters are $\Delta v=0.237 \mathrm{GHz}$, $\mu=2.4$. Other parameters as in Fig. 1(b).

in Figs. 1(b) and 1(c). Moreover, we studied the statistics of the time intervals between two consecutive rogue waves [30,31], and, as can be seen in Fig. 2, the simulated distribution, Fig. 2(b) is in good agreement with the observed one, Fig. 2(a), when a realistic value of the noise strength is included in the simulations.

A precise control of these extreme events requires a good understanding of the mechanism that triggers them. To yield light onto this point, we begin by displaying in Fig. 3(a), for parameters such that rogue waves occur relatively often, the location in phase space of the three fixed points of the model, $\mathrm{S} 1, \mathrm{~S} 2$, and S3.

Figure 3(b) displays a section of a typical trajectory, when no rogue waves occur (in black), and when a rogue wave occurs (in red); Fig. 3(c) displays the onset of the rogue wave. We observe that the trajectory closely approaches the 1D stable manifold of S2 (blue line) and then is repelled: Following the 2D unstable manifold of S2 the trajectory goes far away in phase space, generating the high pulse shown in Fig. 3(d).

Thus, whenever the trajectory closely approaches the stable manifold of S2, a rogue wave is likely to be triggered. Being a deterministic process, it is therefore possible to have some predictability. Typically one needs high-precision information on the trajectory in the 3D phase space; however, as shown in Fig. 1, it is enough to measure the intensity to have a good degree of anticipation for a rogue wave event.

We remark that the trajectory does not need to reach the point S2 for a rogue wave to occur [notice the difference in the vertical scales in Figs. 3(a) and 3(c)]. Before the rogue wave the trajectory follows the stable manifold of $\mathrm{S} 2$, but escapes before reaching $\mathrm{S} 2$, spiraling along the 2D unstable manifold of S2. Since the trajectory does not always escape at the same point in phase space, there is a distribution of rogue wave amplitude values, as was shown in Ref. [24]. Considering this distribution and the chaotic character of the underlying dynamics, the rogue waves in our system cannot be interpreted in terms of excitability.

To understand why the laser intensity displays two types of dynamics, one without rogue waves and one in which they are relatively frequent, we studied the evolution of the distribution 


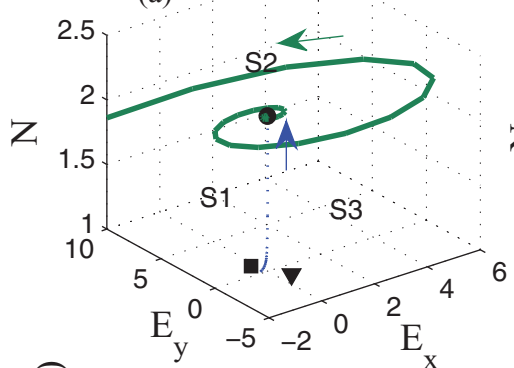

(b)

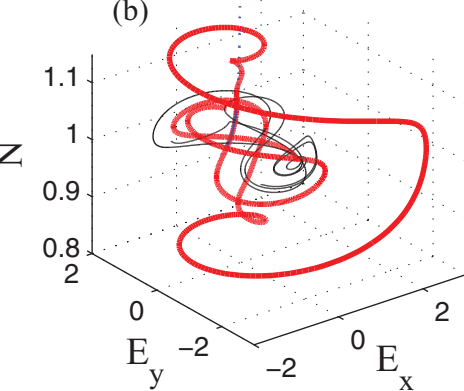

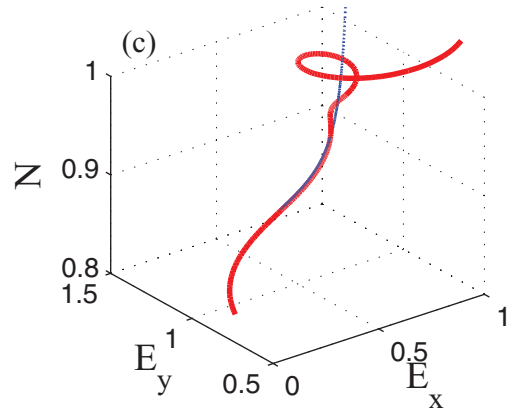

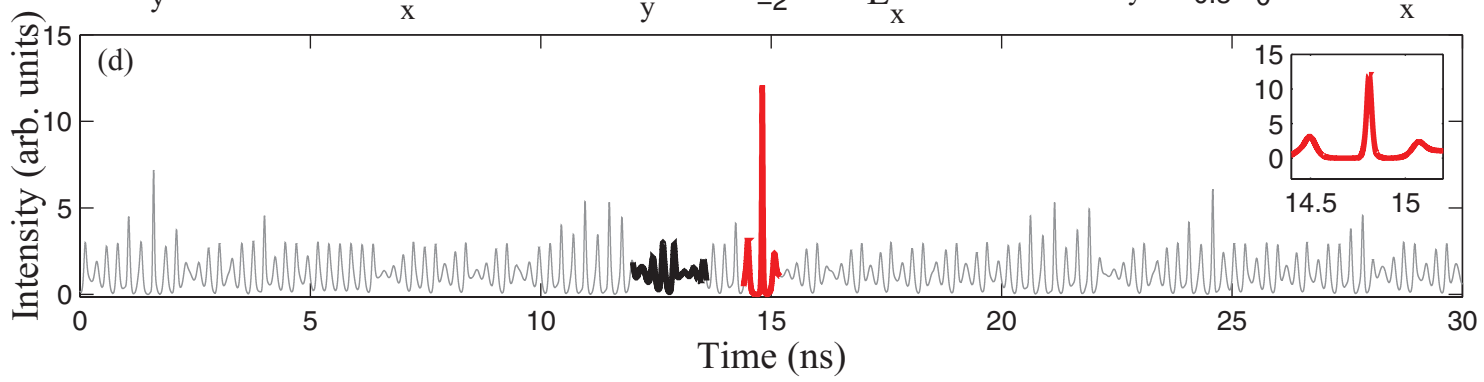

FIG. 3. (Color online) (a) Phase space representation of the model fixed points, S1, S2, and S3. S1 is a saddle; S2 and S3 are unstable foci. The lines dotted (blue online) and solid (green online) indicate the stable and unstable manifolds of S2. (b) Typical trajectory (back thin line) in the dense part of the chaotic attractor, and, in the thick line (red online), the trajectory during a visit to the sparse part of the attractor, when a rogue wave occurs. The dotted line (blue online) indicates the stable manifold of S2. (c) Detail of the onset of the rogue wave, where one can observe that the trajectory closely approaches the stable manifold of S2 before the rogue wave occurs. (d) Time trace displaying a rogue event: The thick segments (rogue event expanded in the inset) correspond to the trajectory segments in (b). The parameters are as in Fig. 1(b).

of the intensity pulse amplitude, when a control parameter changes.

Figure 4 presents the experimental distribution and the optical spectrum when the VCSEL bias current is varied. Due to Joule heating, the detuning between the injected and master laser also varies from $17 \mathrm{GHz}(0.4 \mathrm{~mA})$ to $-18 \mathrm{GHz}$ $(0.72 \mathrm{~mA})$ [32]. The injected VCSEL and the master laser do not interact for very large (positive or negative) detuning values (below $0.44 \mathrm{~mA}$ and above $0.69 \mathrm{~mA}$ ) while they are locked at 0.47 up to $0.51 \mathrm{~mA}$. Rogue waves were found in current regions between 0.62 and $0.64 \mathrm{~mA}$, as shown by the tails of the histograms, which cross the rogue waves threshold (red line) by a wide margin.

In the numerical bifurcation diagram [Fig. 5(a)] rogue waves are seen only in a very narrow parameter region, just when the chaotic attractor that develops from the fixed point S3 suffers a sudden expansion. As shown in Figs. 5(b) and 5(c) that display a Poincare section of the attractor, this sudden expansion is the result of an external crisis-like process that is generated by the crossing of the attractor with the stable manifold of S1. In Fig. 5(b) we observe that the attractor is "tangent" in several points to this manifold (red line). We remark that this crisis process differs from the most common type of crisis, referred to as an interior crisis [27], that results in a progressive gradual expansion of the chaotic attractor in phase space.

After the crossing the trajectory can approach the region of the phase space where the stable manifold of S2 is (blue cross), and each time the trajectory closely reaches this region, a high-intensity pulse will be triggered.

We remark that in the simulations without noise we have not seen rogue waves for parameters before the crossing occurs; however, when noise is included, a few rogue waves might be seen. The presence of noise can generate extremely high pulses in unidirectionally coupled chaotic systems [33], and in our case, it anticipates the general dynamical behavior; thus extreme pulses are possible even for parameters before the crossing actually occurs. In this sense, we summarize the role of noise in our system as the following:

(i) In parameter regions where deterministic rogue waves occur, the inclusion of noise is "detrimental" for rogue waves because it diminishes the probability of the trajectory finding the narrow region in phase space (in the very close vicinity of the stable manifold of S2) that the trajectory needs to approach in order to start a rogue wave (we refer to this region as "the narrow rogue wave door"). In the absence of noise, the deterministic dynamics tends to take the system there, and when noise is included this region is harder to find.

(ii) On the other hand, for parameters close to the crossing, where there are no deterministic rogue waves, noise is "beneficial" for triggering them, as it helps in finding the rogue wave door that exists, but, because the crossing did not yet occur, the deterministic dynamics does not take the system close to this region, and thus noise helps the system overcoming the "S1 barrier," and then the system can reach "the narrow rogue wave door."

To conclude, we studied experimentally and numerically optical rogue waves in an injected semiconductor laser and demonstrated that the giant intensity pulses can be anticipated and that their probability of occurrence can be reduced when noise is included. We also demonstrated that the mechanism generating these extreme intensity pulses is an external crisis-like process in the form of a crossing of the attractor generated from one fixed point with the stable manifold 

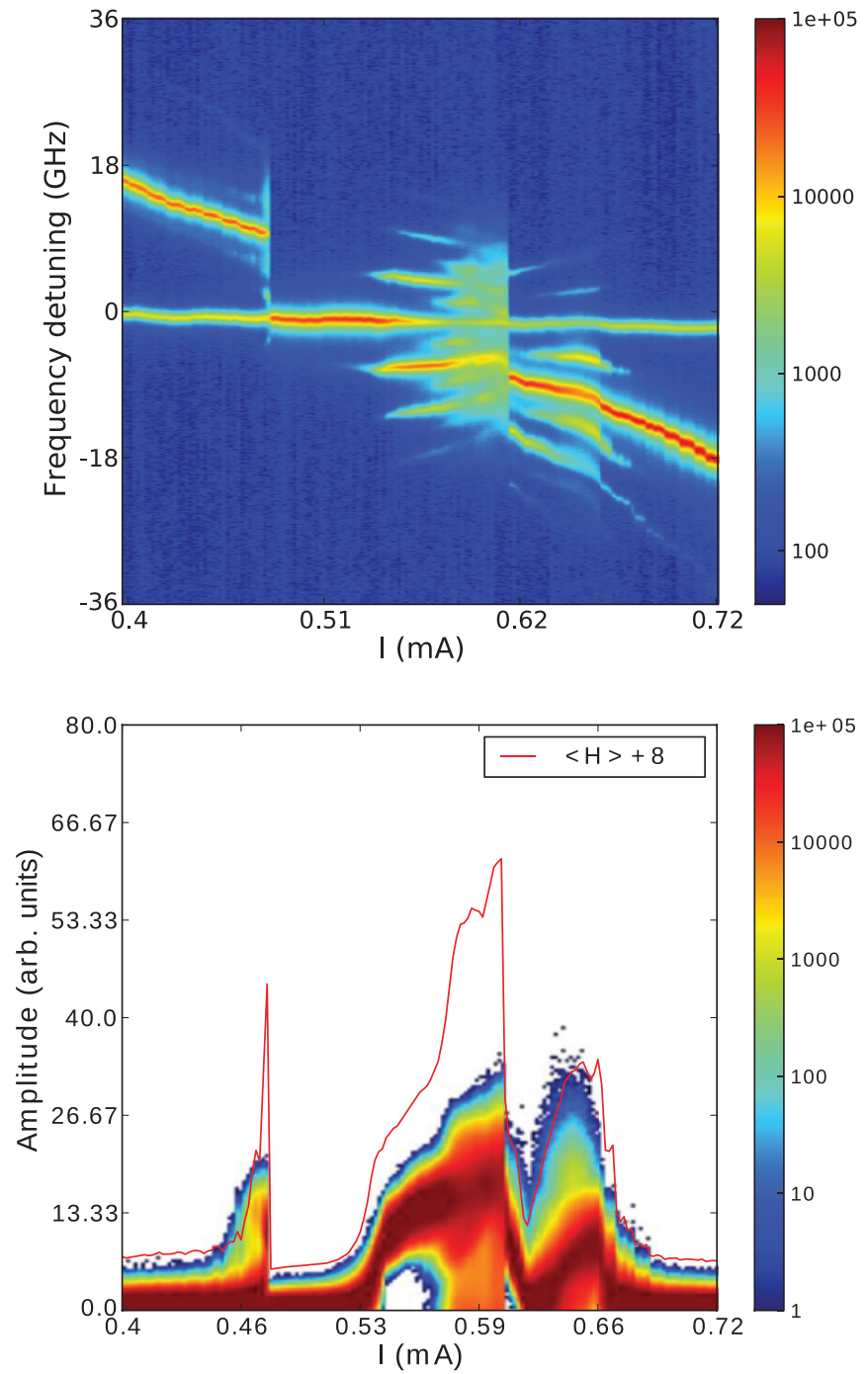

FIG. 4. (Color online) Experimental optical spectrum (a) and wave amplitude distribution (b) when varying the VCSEL bias current (logarithmic scale). The injected power is $21 \mu \mathrm{W}$, and the VCSEL bias current (whose threshold is about $0.2 \mathrm{~mA}$ ) is varied from 0.4 to $0.72 \mathrm{~mA}$ in steps of $1 \mu \mathrm{A}$. Histograms of the wave amplitudes are calculated over $400 \mu \mathrm{s}$ long-time series which contain up to $10^{6}$ waves. The line (red online) denotes the rogue wave threshold; pulses surpassing this limit occur between 0.62 and $0.64 \mathrm{~mA}$.

of another fixed point. Since this phase space structure (attractor/barrier/saddle) occurs in many dissipative nonlinear
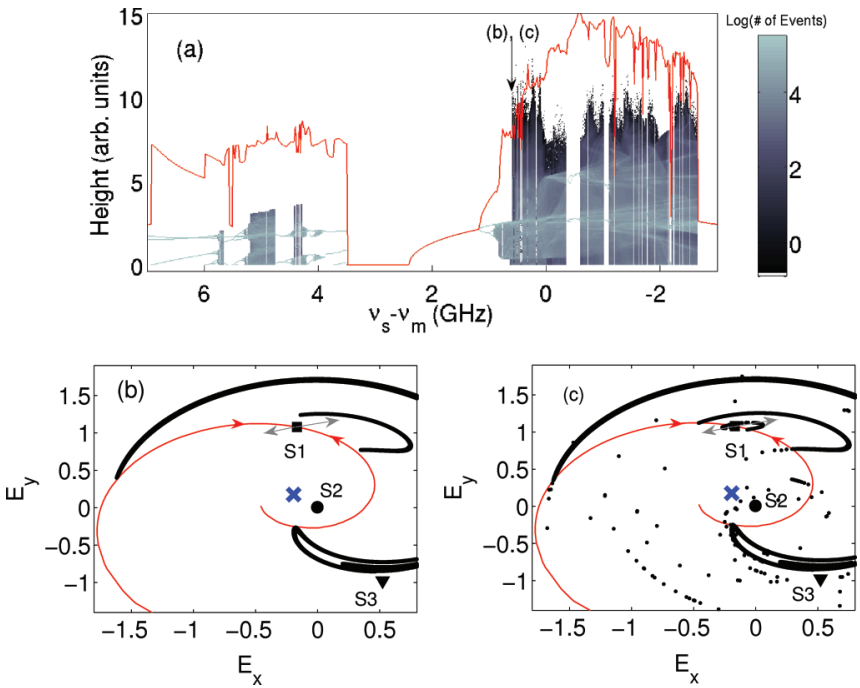

FIG. 5. (Color online) (a) Numerical bifurcation diagram displaying the height of the oscillations vs the frequency detuning. The gray color code indicates the number of oscillations at a given height, and the line (red online) the threshold that defines a rogue wave. The parameters are as in Fig. 1(b). The horizontal axis shows $v_{m}-v_{s}$ for easier comparison with the experimental diagram (as an increase of the bias current of the injected laser decreases its optical frequency). (b, c) Poincaré section of the attractor obtained by plotting the points where the trajectory crosses the plane that contains the saddle point $\mathrm{S} 1$ (the intersection with the plane $N=1.0036$ ) for $v_{s}-v_{m}=0.6$ and $0.594 \mathrm{GHz}$, respectively. In this plane we observe the collision of the chaotic attractor (in black) with the stable manifold of S1 (gray line, red online). The arrows indicate the direction of movement along the manifolds of S1. After the collision, in panel (c) trajectories can access the region of phase space where the stable manifold of S2 is found (indicated by a cross), and a rogue wave occurs whenever the trajectory closely visits this region. For easier comparison with Fig. 3, $\mathrm{S} 2$ and $\mathrm{S} 3$ are projected in the Poincaré plane.

dynamical systems, our results have wide implications. Crises are common in multistable systems [19], and they are likely to produce rare and extreme events.

J.Z.M. acknowledges useful discussions with M. Matias and support from the grant FISICOS of the Spanish MCI (FIS2007-60327). J.R.R.L. acknowledges Brazilian FACEPECNPQ-Pronex-APQ0630-1.05-06. C.M. acknowledges partial support from EOARD US (FA-8655-10-1-3075), the Spanish MCI (FIS2009-13360), the Generalitat de Catalunya (2009 SGR 1168), and the ICREA Academia program.
[1] M. S. Longuet-Higgins, Philos. Trans. R. Soc. London A 249, 321 (1957).

[2] A. N. Ganshin, V. B. Efimov, G. V. Kolmakov, L. P. Mezhov-Deglin, and P. V. E. McClintock, Phys. Rev. Lett. 101, 065303 (2008).

[3] V. B. Efimov, A. N. Ganshin, G. V. Kolmakov, P. V. E. McClintock, and L. P. Mezhov-Deglin, Eur. Phys. J. Sel. Top. 185, 181 (2010).
[4] Z. Y. Yan, Commun. Theor. Phys. 54, 947 (2010).

[5] W. X. Wang, R. Yang, Y. C. Lai, V. Kovanis, and C. Grebogi, Phys. Rev. Lett. 106, 154101 (2011).

[6] S. Aberg and G. Lindgren, Probab. Eng. Mech. 23, 359 (2008).

[7] A. Chabchoub, N. P. Hoffmann, and N. Akhmediev, Phys. Rev. Lett. 106, 204502 (2011).

[8] M. Onorato, D. Proment, and A. Toffoli, Phys. Rev. Lett. 107, 184502 (2011). 
[9] A. Chabchoub, N. Hoffmann, M. Onorato, and N. Akhmediev, Phys. Rev. X 2, 011015 (2012).

[10] D. R. Solli, C. Ropers, P. Koonath, and B. Jalali, Nature (London) 450, 1054 (2007).

[11] J. M. Dudley, G. Genty, and B. J. Eggleton, Opt. Express 16, 3644 (2008).

[12] J. Kasparian, P. Bejot, J.-P. Wolf, and J. M. Dudley, Opt. Express 17, 12070 (2009).

[13] A. Mussot, A. Kudlinski, M. Kolobov, E. Louvergneaux, M. Douay, and M. Taki, Opt. Express 17, 17010 (2009).

[14] N. Akhmediev, J. M. Soto-Crespo, and A. Ankiewicz, Phys. Lett. A 373, 2137 (2009).

[15] N. Akhmediev, Jose M. Soto-Crespo, A. Ankiewicz, and N. Devine, Phys. Lett. A 375, 2999 (2011).

[16] U. Bandelow and N. Akhmediev, Phys. Lett. A 376, 1558 (2012).

[17] F. T. Arecchi, U. Bortolozzo, A. Montina, and S. Residori, Phys. Rev. Lett. 106, 153901 (2011).

[18] F. M. de Aguiar, Phys. Lett. A 375, 265 (2011).

[19] A. N. Pisarchik, R. Jaimes-Reategui, R. Sevilla-Escoboza, G. Huerta-Cuellar, and M. Taki, Phys. Rev. Lett. 107, 274101 (2011).

[20] S. Randoux and P. Suret, Opt. Lett. 37, 500 (2012).

[21] M. Kovalsky, A. A. Hnilo, and J. R. Tredicce, Opt. Lett. 36, 4449 (2011).

[22] A. Zaviyalov, O. Egorov, R. Iliew, and F. Lederer, Phys. Rev. A 85, 013828 (2012).

[23] C. Lecaplain, Ph. Grelu, J. M. Soto-Crespo, and N. Akhmediev, Phys. Rev. Lett. 108, 233901 (2012).
[24] C. Bonatto, M. Feyereisen, S. Barland, M. Giudici, C. Masoller, Jose R. Rios Leite, and J. R. Tredicce, Phys. Rev. Lett. 107, 053901 (2011).

[25] F. Baronio, A. Degasperis, M. Conforti, and S. Wabnitz, Phys. Rev. Lett. 109, 044102, (2012).

[26] J. M. Dudley, C. Finot, G. Millot, J. Garnier, G. Genty, D. Agafontsev, and F. Dias, Eur. Phys. J. Sel. Top. 185, 125 (2010).

[27] R. Gilmore and M. Lefranc, The Topology of Chaos, Alice in Stretch and Squeezeland (John Wiley, New York, 2002).

[28] C. Grebogi, E. Ott, and J. A. Yorke, Physica D 7, 181 (1983).

[29] Both in the experimental and in the simulated intensity time traces, rogue wave events are considered as pulses whose amplitude is above a threshold that is defined as the average pulse height, $\langle H\rangle$ plus $X$ times the standard deviation of the distribution of pulse heights, $\sigma$. As in Ref. [24] we chose $X=8$ except in Fig. 1(d) where we use $X=4$ to show the difference in the anticipation time of $X=8$ and $X=4$ rogue waves, and in Fig. 2, where we use $X=6$ to have enough statistics in a single time trace.

[30] C. Nicolis and S. C. Nicolis, Eur. Phys. Lett. 80, 40003 (2007).

[31] C. Nicolis and G. Nicolis, Phys. Rev. E 80, 061119 (2009).

[32] As compared to Ref. [24], we are able to work at lower bias current thanks to an improved detection system.

[33] S. C. Venkataramani, B. R. Hunt, E. Ott, D. J. Gauthier, and J. C. Bienfang, Phys. Rev. Lett. 77, 5361 (1996). 